\title{
Neonatal Outcomes and their Relationship to Maternal Buprenorphine Dose during Pregnancy
}

\author{
Hendrée E. Jones ${ }^{a, b}$, Erin Dengler ${ }^{c}$, Anna Garrisond ${ }^{d}$, Kevin E. O'Grady ${ }^{e}$, Carl Seashore ${ }^{f}$, \\ Evette Horton $^{\mathrm{a}}$, Kim Andringa ${ }^{\mathrm{a}}$, Lauren M. Jansson ${ }^{\mathrm{g}}$, and John Thorp ${ }^{\mathrm{a}}$ \\ aUNC Horizons and Department of Obstetrics and Gynecology, University of North Carolina at \\ Chapel Hill, Carrboro, NC 27510 \\ bDepartments of Psychiatry and Behavioral Sciences and Obstetrics and Gynecology, School of \\ Medicine, Johns Hopkins University, Baltimore, MD 21224 \\ 'Department of Neuroscience, Washington and Lee University, Lexington, VA 24450 \\ dDepartment of Sociology, University of North Carolina at Chapel Hill, Chapel Hill, NC 27514 \\ eDepartment of Psychology, University of Maryland, College Park, College Park, MD 20742 \\ fDepartment of Pediatrics, University of North Carolina at Chapel Hill, Chapel Hill, NC 27514 \\ gDepartment of Pediatrics, School of Medicine, Johns Hopkins University, Baltimore, MD 21224
}

\section{Abstract}

Background-Buprenorphine pharmacotherapy for opioid-dependent pregnant women is associated with maternal and neonatal outcomes superior to untreated opioid dependence. However, the literature is inconsistent regarding the possible existence of a dose-response relationship between maternal buprenorphine dose and neonatal clinical outcomes.

Methods-The present secondary analysis study (1) examined the relationship between maternal buprenorphine dose at delivery and neonatal abstinence syndrome (NAS) peak score, estimated gestational age at delivery, Apgar scores at 1 and 5 minutes, neonatal head circumference, length, and weight at birth, amount of morphine needed to treat NAS, duration of NAS treatment, and duration of neonatal hospital stay; and (2) compared neonates who required pharmacotherapy for NAS to neonates who did not require such pharmacotherapy on these same outcomes, in 58 opioid-dependent pregnant women receiving buprenorphine as participants in a randomized clinical trial.

Results-(1) Analyses failed to provide evidence of a relationship between maternal buprenorphine dose at delivery and any of the 10 outcomes (all $p$-values $>.48$ ); and (2) significant

(C) 2013 Elsevier Ireland Ltd. All rights reserved.

TO: Corresponding Author: Hendrée E. Jones UNC Horizons Department of Obstetrics and Gynecology University of North Carolina at Chapel Hill 127 Kingston Drive Chapel Hill, NC 27514 USA Voice: 1-919-966-9803 Fax: 1-919-966-9169 hendree_jones@med.unc.edu.

Publisher's Disclaimer: This is a PDF file of an unedited manuscript that has been accepted for publication. As a service to our customers we are providing this early version of the manuscript. The manuscript will undergo copyediting, typesetting, and review of the resulting proof before it is published in its final citable form. Please note that during the production process errors may be discovered which could affect the content, and all legal disclaimers that apply to the journal pertain. 
mean differences between the untreated $(n=31)$ and treated $(n=27)$ for NAS groups were found for duration of neonatal hospital stay and NAS peak score (both $p$-values<.001).

Conclusions-(1) Findings failed to support the existence of a dose-response relationship between maternal buprenorphine dose at delivery and any of 10 neonatal clinical outcomes, including NAS severity; and (2) that infants treated for NAS had a higher mean NAS peak score and, spent a longer time in the hospital than did the group not treated for NAS is unsurprising.

\section{Keywords}

opioid use disorder; pregnancy; buprenorphine; neonate; neonatal abstinence syndrome

\section{INTRODUCTION}

Research has strongly suggested that buprenorphine pharmacotherapy for pregnant women with opioid use disorder is associated with superior maternal and neonatal outcomes relative to untreated opioid use disorder. Moreover, in terms of the relative efficacy of buprenorphine compared to methadone during pregnancy, Jones et al. (2012b) presented a systematic review of the literature in which they reported that buprenorphine and methadone have comparable maternal efficacy, that buprenorphine may produce less physiological suppression of fetal heart rate and movements than methadone, and that exposure in utero to buprenorphine results in a less severe neonatal abstinence syndrome (NAS) than methadone. A focus on NAS is of considerable current importance because its incidence in the US has increased from 1.2 to 3.4 per 1000 hospital live births from 2000 to 2009. During this same period, mean hospital charges for treatment of neonates with NAS increased more than $35 \%$, from $\$ 39,400$ to $\$ 53,400$ (Patrick et al., 2012).

However, the relationship between maternal buprenorphine dose and either neonatal abstinence syndrome (NAS) incidence or severity has been inconsistent (Jones et al., 2005; Lejeune et al., 2006), a finding also reported for methadone (Jones et al., 2013; O'Grady et al., 2013). Infant urinary concentrations of norbuprenorphine, the primary buprenorphine metabolite, have been found to correlate with infant length of hospital stay but not duration of NAS pharmacotherapy (Hytinantti et al., 2008). In line with this finding, total buprenorphine concentrations in meconium and buprenorphine/norbuprenorphine ratios were associated with the presence of a diagnosable NAS, although not necessarily one requiring pharmacotherapy (Kacinko et al., 2008). Examination of the possibility of such a dose-response relationship between maternal dose of buprenorphine and neonatal outcomes including NAS severity is of considerable clinical importance, because determination of the existence of such a relationship would have implications for the medical management of pregnant women with opioid use disorder. Such a finding would suggest the potential need to limit or restrict the buprenorphine dose for the mother in order to reduce the deleterious impact of a higher dose on the neonate. Such a restriction could be potentially disadvantageous to the treatment of the mother if the dose was insufficient to ameliorate or reduce illicit opioid use or licit opioid misuse. Moreover, knowing the extent to which buprenorphine-exposed neonates who require pharmacotherapy for NAS differ from buprenorphine-exposed neonates who do not require pharmacotherapy for NAS on clinically 
relevant neonatal outcomes would provide information that could be used to guide the medical management of NAS.

Research on the relationship between buprenorphine dose and neonatal clinical outcomes has largely although not entirely focused on the differences between buprenorphine and methadone in NAS occurrence or severity, and to a lesser extent on morphine dose to treat NAS, and length of hospitalization for NAS treatment. Research on the relationship between buprenorphine dose and severity of NAS has typically failed to find any such relationship (Bakstad et al., 2009; Fischer et al., 2006; Kacinko et al., 2008, Lejeune et al., 2006; O'Connor et al., 2011). Metz et al. (2011) also reported a failure to find relationships between buprenorphine dose and need for and amount of NAS medication, peak NAS score, and duration of NAS treatment in a sample of 26 neonates prenatally exposed to buprenorphine as part of comprehensive treatment program for maternal opioid use disorder.

The purpose of this secondary analysis study was twofold: (1) to examine the dose-response relationship between maternal buprenorphine and a variety of neonatal clinical outcomes, and (2) to compare neonates who require pharmacotherapy for NAS to neonates who do not require such pharmacotherapy on these same outcomes, in a sample of 58 mothers with opioid use disorders who participated in a randomized clinical trial of opioid-agonist pharmacotherapy.

\section{METHODS}

\subsection{The Maternal Opioid Treatment: Human Experimental Research (MOTHER) Study}

MOTHER (Jones et al., 2012a, 2010) was a double-blind, double-dummy, flexible-dosing, parallel-group randomized clinical trial comparing outcomes for pregnant women with opioid use disorders and their neonates receiving either buprenorphine or methadone pharmacotherapy provided in the context of comprehensive care. Study findings showed that, on average, neonates in the buprenorphine condition compared to neonates in the methadone condition required significantly less morphine to treat NAS, has a shorter NAS treatment course, and spent significantly less time in the hospital. Details about the MOTHER study necessary to understanding the current analyses follow. More complete information about MOTHER is available in Jones et al. (2010, 2012).

\subsection{Participants}

One-hundred-seventy-five pregnant women with opioid use disorders meeting eligibility criteria participated in the study. Women were randomly assigned to either the buprenorphine or the methadone condition in which double-blind, double-dummy, study medication was dispensed daily with sublingual tablets (buprenorphine or placebo) followed by oral liquid (methadone or placebo). The buprenorphine condition utilized a flexible dose range of 2-32 mg. Concomitant drug use was reduced through the use of monetary vouchers provided to participants for providing three-times-weekly urine samples testing negative for opioids (other than their study medication) and other illicit or non-prescribed drugs. Onehundred-thirty-one of the 175 maternal participants delivered neonates while enrolled in the 
study, of whom 58 were in the buprenorphine condition. The secondary analyses reported below use the data from the neonates of these 58 maternal participants.

\subsection{Neonatal Outcomes}

2.3.1 Neonatal Abstinence Syndrome (NAS)_All infants were hospitalized for a minimum of 4 days for observation for the development of NAS. Regardless of in-patient or out-patient status, neonates were assessed for NAS by trained staff for a minimum of 10 days using a modified Finnegan Scale. Oral morphine sulfate was used for NAS treatment. All pharmacotherapy for NAS was delivered to the infants during an inpatient hospital stay; no infant received medication for the treatment of NAS as an outpatient. A NAS peak score, the highest NAS score the neonate obtained during this period, was calculated for each neonate.

2.3.2 Other Neonatal Outcomes-Additional neonatal outcomes included estimated gestational age at delivery (weeks), Apgar scores at 1 and 5 minutes, neonatal head circumference $(\mathrm{cm})$, length $(\mathrm{cm})$, and weight at birth $(\mathrm{g})$, total amount of morphine needed to treat NAS (mg), duration of treatment for NAS (days), and duration of neonatal hospital stay (days). See Jones et al. (2010) for detailed descriptions of all measures.

\subsection{Statistical Analyses}

As in the primary outcomes paper (Jones et al., 2010), total amount of morphine needed to treat NAS (mg), infant length of stay in the hospital (days), number of days medicated for NAS (days), estimated gestational age at delivery (weeks), and Apgar scores at 1 and 5 minutes were analyzed with Poisson regression, allowing for overdispersion. Peak score on the MOTHER NAS scale during the assessment period, and infant head circumference, birth weight, and length were analyzed with ordinary least squares regression. Maternal buprenorphine dose $(\mathrm{mg})$ at delivery, collected as part of the MOTHER trial, and site of MOTHER data collection (US Urban [Baltimore, MD; Philadelphia, PA; Detroit MI; Providence, RI] $v$. US Rural [Burlington, VT; Nashville, TN] $v$. European [Vienna]) served as the explanatory variables in all analyses.

\section{RESULTS}

Maternal buprenorphine dose at delivery was 4-32 $\mathrm{mg}(M=16.6, S D=7.3)$. Table 1 contains the descriptive statistics as well as the parameter estimates and standard errors associated with the analyses of the 10 neonatal outcomes. Analyses failed to provide any evidence of a relationship between maternal buprenorphine dose at delivery and the respective outcome measure (all $p \mathrm{~s}>.48$ ).

For those infants requiring pharmacotherapy for NAS, the mean total amount of morphine (mg) was $3.5(S D=3.5)$, while the mean duration of treatment for NAS was 9.8 days $(S D=5.5)$. Significant mean differences between the untreated $(n=31)$ and treated $(n=27)$ for NAS groups were found for duration of neonatal hospital stay, $F(1,54)=15.5, p<.001,[M$ $=6.5$ days $(S E=1.0)$ v. $M=14.1$ days $(S E=1.6)$, respectively] and NAS peak score, $F(1$, $53)=66.3, p<.001,[M=8.5(S E=0.4) v . M=13.9(S E=0.4)$, respectively $]$. All other 
tests of mean differences between the NAS treatment status groups were nonsignificant (all $p$-values > .4).

\section{DISCUSSION}

This secondary analysis study of data from the MOTHER trial failed to support any relationship between maternal buprenorphine dose at delivery and any of a number of clinically important neonatal outcomes. There was no relationship between maternal buprenorphine dose at delivery and NAS severity, as measured by peak NAS score, total amount of morphine needed to treat NAS, duration of treatment for NAS, or duration of neonatal hospital stay, or with any of 6 other neonatal clinical outcomes, including estimated gestational age at delivery, Apgar scores at 1 and 5 minutes, neonatal head circumference, length, and weight at birth. These results are consistent with and expand upon previously reported findings (Jones et al., 2013), in which no significant relationship was found between maternal methadone dose and neonatal outcome. Moreover, the failure to find a dose-response relationship expand between buprenorphine and duration of neonatal hospital stay, estimated gestational age at delivery, Apgar scores at 1 and 5 minutes, neonatal head circumference, length, and weight at birth expand upon the findings of Metz et al. (2011).

In contrast to many of the previous studies of the relationship between buprenorphine dose and NAS severity, in the present study the failure to find a relationship between buprenorphine dose and neonatal clinical outcomes occurred in a sample in which the concomitant substance use that might serve to confound such relationships was negligible or non-existent, participants had neither alcohol or benzodiazepine use disorders, and minimal use of cocaine and other substances. Thus, the present sample permitted assessment of the relationship between buprenorphine dose and neonatal outcomes without the potentially confounding factor of poly-substance use. Moreover, the assessment of NAS was undertaken with a validated instrument with which all raters had been trained, and on which they received episodic re-training. And, the NAS medication criteria were well-defined and uniformly applied.

That the infants treated for NAS had a higher mean NAS peak score and, on average, spent a longer time in the hospital than did the group not treated for NAS is unsurprising. Both of these outcomes are likely the result from a more severe NAS presentation and need for greater pharmacotherapy for these infants.

The failure to find a dose-response relationship between maternal buprenorphine dose and any of a number of neonatal clinical outcomes in a well-controlled clinical trial is an important result. The use of any medication has fundamental benefits and risks. This circumstance is particularly true with pharmacotherapy for opioid use disorder in pregnant women, in whom the risks as well as the benefits are likely to be of a large magnitude. There remains a persistent misunderstanding that limiting the exposure of the fetus to maintenance medications for the treatment of maternal opioid use disorder is in the best interests of both the mother and neonate. In these dyads, wellness of the mother is inherently tied to infant outcomes. Maternal abstinence from illicit opioid use, which is necessarily accomplished in many such mothers by maintenance on an appropriate dose of a medication such as 
methadone or buprenorphine, leading to improved access to prenatal care and subsequent avoidance of myriad medical and psychosocial complications of continued drug use, are of profound importance to the infant. The present findings, together with those of Jones et al. (2013), lend support to the belief that there is no sound basis for limiting the dose of opioid agonist medication provided to pregnant women with opioid use disorder in order to minimize its impact on the neonate. Future research needs to examine the relationship between buprenorphine dose and neonatal clinical outcomes in other samples of neonates that are more diverse in terms of such factors as their exposure to other illicit substances, and/or whose mothers are not in comprehensive care for their opioid use disorder. Finally, more research needs to be conducted regarding the longer-term effects of in utero exposure to opioid agonist and mixed agonist/antagonist medications.

\section{REFERENCES}

Bakstad B, Sarfi M, Welle-Strand GK, Ravndal E. Opioid maintenance treatment during pregnancy: occurrence and severity of neonatal abstinence syndrome. A national prospective study. Eur. Addict. Res. 2009; 15:128-134. [PubMed: 19332991]

Fischer G, Ortner R, Rohrmeister K, Jagsch R, Baewert A, Langer M, Aschauer H. Methadone versus buprenorphine in pregnant addicts: a double-blind, double-dummy comparison study. Addiction. 2006; 101:275-281. [PubMed: 16445556]

Hytinantti T, Kahila H, Renlund M, Jarvenpaa AL, Halmesmaki E, Kivitie-Kallio S. Neonatal outcome of 58 infants exposed to maternal buprenorphine in utero. Acta Paediatr. 2008; 97:1040-1044. [PubMed: 18474065]

Jones HE, Fischer G, Heil SH, Kaltenbach K, Martin PR, Coyle MG, Selby P, Stine SM, O'Grady KE, Arria AM. Maternal Opioid Treatment: Human Experimental Research (MOTHER): approach, issues, and lessons learned. Addiction. 2012a; 107(Suppl. 1):28-35. [PubMed: 23106924]

Jones HE, Heil SH, Baewert A, Arria AM, Kaltenbach K, Martin PR, Coyle MG, Selby P, Stine SM, Fischer G. Buprenorphine treatment of opioid-dependent pregnant women: a comprehensive review. Addiction. 2012b; 107(Suppl. 1):5-27. [PubMed: 23106923]

Jones HE, Jansson LM, O'Grady KE, Kaltenbach K. The relationship between maternal methadone dose at delivery and neonatal outcome: methodological and design considerations. Neurotoxicol. Teratol. 2013; 39:110-115. [PubMed: 24099621]

Jones HE, Johnson RE, Jasinski DR, O'Grady KE, Chisholm CA, Choo RE, Crocetti M, Dudas R, Harrow C, Huestis MA, Jansson LM, Lantz M, Lester BM, Milio L. Buprenorphine versus methadone in the treatment of pregnant opioid-dependent patients: effects on the neonatal abstinence syndrome. Drug Alcohol Depend. 2005; 79:1-10. [PubMed: 15943939]

Jones HE, Kaltenbach K, Heil SH, Stine SM, Coyle MG, Arria AM, O'Grady KE, Selby P, Martin PR, Fischer G. Neonatal abstinence syndrome after methadone or buprenorphine exposure. N. Engl. J. Med. 2010; 363:2320-2331. [PubMed: 21142534]

Kacinko SL, Jones HE, Johnson RE, Choo RE, Huestis MA. Correlations of maternal buprenorphine dose, buprenorphine, and metabolite concentrations in meconium with neonatal outcomes. Clin. Pharmacol. Ther. 2008; 84:604-612. [PubMed: 18701886]

Lejeune C, Simmat-Durand L, Gourarier L, Aubisson S. Prospective multicenter observational study of 260 infants born to 259 opiate-dependent mothers on methadone or high-dose buprenophine substitution. Drug Alcohol Depend. 2006; 82:250-257. [PubMed: 16257138]

Metz V, Jagsch R, Ebner N, Wurzl J, Pribasnig A, Aschauer C, Fischer G. Impact of treatment approach on maternal and neonatal outcome in pregnant opioid-maintained women. Hum. Psychopharmacol. 2011; 26:412-421. [PubMed: 21823171]

O'Connor A, Alto W, Musgrave K, Gibbons D, Llanto L, Holden S, Karnes J. Observational study of buprenorphine treatment of opioid-dependent pregnant women in a family medicine residency: reports on maternal and infant outcomes. J. Am. Board Fam. Med. 2011; 24:194-201. [PubMed: 21383220] 
O'Grady KE, Jones HE, Jansson LM, Kaltenbach K. Response to Wouldes and Woodward: maternal methadone dose during pregnancy and infant clinical outcome. Neurotoxicol. Teratol. 2013; 39:116-118. [PubMed: 23416427]

Patrick SW, Schumacher RE, Benneyworth BD, Krans EE, McAllister JM, Davis MM. Neonatal abstinence syndrome and associated health care expenditures: United States, 2000-2009. JAMA. 2012; 307:1934-1940. [PubMed: 22546608] 


\section{Table 1}

Total Sample Means and (Standard Deviations) and Parameter Estimates from the Regression Analyses Examining the Relationship between Buprenorphine Dose and 10 Neonatal Clinical Outcomes $(\mathrm{N}=58)$

\begin{tabular}{|c|c|c|c|c|c|c|}
\hline Outcome Variable & Mean (SD) & b & SE & AOR & $95 \% \mathrm{CI}$ & $\mathbf{p}$ \\
\hline Total amount of morphine for $\mathrm{NAS}^{\dagger}(\mathrm{mg})$ & $1.7(3.0)$ & & & .98 & $.90,1.07$ & .70 \\
\hline Duration of treatment for $\mathrm{NAS}^{\dagger}$ (days) & $4.6(6.2)$ & & & .99 & $.92,1.06$ & .78 \\
\hline Duration of neonatal hospital stay (days) & $10.1(7.0)$ & & & .99 & $.97,1.03$ & .95 \\
\hline Gestational age at delivery (weeks) & $39.1(2.2)$ & & & 1.00 & $.99,1.00$ & .82 \\
\hline Apgar score at 1 minute & $8.1(1.6)$ & & & 1.00 & $.99,1.02$ & .86 \\
\hline Apgar score at 5 minutes & $9.0(1.1)$ & & & 1.00 & $.99,1.01$ & .99 \\
\hline NAS peak score ${ }^{\dagger}$ & $11.0(3.4)$ & -.05 & .07 & & & .50 \\
\hline Neonatal head circumference at birth $(\mathrm{cm})^{\dagger}$ & $33.8(1.9)$ & .01 & .04 & & & .78 \\
\hline Neonatal weight at birth (g) & $3096.9(561.2)$ & 1.34 & 11.93 & & & .91 \\
\hline Neonatal length at birth $(\mathrm{cm})^{\dagger}$ & $49.8(2.7)$ & .04 & .06 & & & .48 \\
\hline
\end{tabular}

Notes. Means do not equal values found in Table 2 of Jones et al. (2010), because the latter means are model-derived marginal means from a model that includes site and the methadone condition. SD $=$ Standard Deviation. NAS $=$ Neonatal Abstinence Syndrome. $\mathrm{b}=$ unstandardized partial regression coefficient. SE $=$ Standard Error. AOR = Adjusted Odds Ratio (adjusted for the fixed site factor), $95 \% \mathrm{CI}=95 \% \mathrm{Confidence}$ Interval

${ }^{\dagger}$ One case is missing data for this variable. 